\title{
Characterization of the minimum energy paths for the ring closure reactions of $\mathrm{C}_{4} \mathrm{H}_{3}$ with acetylene
}

\author{
Stephen P. Walch \\ Thermosciences Institute, NASA Ames Research Center, Moffett Field, California 94035-1000
}

(Received 23 March 1995; accepted 14 August 1995)

\begin{abstract}
The ring closure reaction of $\mathrm{C}_{4} \mathrm{H}_{3}$ with acetylene to give phenyl radical is one proposed mechanism for the formation of the first aromatic ring in hydrocarbon combustion. There are two low-lying isomers of $\mathrm{C}_{4} \mathrm{H}_{3} ;$ 1-dehydro-buta-1-ene-3-yne $\left(n-\mathrm{C}_{4} \mathrm{H}_{3}\right)$ and 2-dehydro-buta-1-ene-3-yne (iso- $\mathrm{C}_{4} \mathrm{H}_{3}$ ). It has been proposed that only $n-\mathrm{C}_{4} \mathrm{H}_{3}$ reacts with acetylene to give phenyl radical, and since iso- $\mathrm{C}_{4} \mathrm{H}_{3}$ is more stable than $n-\mathrm{C}_{4} \mathrm{H}_{3}$, formation of phenyl radical by this mechanism is unlikely. We report restricted Hartree-Fock (RHF) plus singles and doubles configuration interaction calculations with a Davidson's correction (RHF+1+2+Q) using the Dunning correlation consistent polarized valence double zeta basis set (cc-pVDZ) for stationary point structures along the reaction pathway for the reactions of $n-\mathrm{C}_{4} \mathrm{H}_{3}$ and iso- $\mathrm{C}_{4} \mathrm{H}_{3}$ with acetylene. $n-\mathrm{C}_{4} \mathrm{H}_{3}$ plus acetylene (9.4) has a small entrance channel barrier (17.7) (all energetics in parentheses are in $\mathrm{kcal} / \mathrm{mol}$ with respect to iso- $\mathrm{C}_{4} \mathrm{H}_{3}$ plus acetylene) and the subsequent closure steps leading to phenyl radical $(-91.9)$ are downhill with respect to the entrance channel barrier. Iso- $\mathrm{C}_{4} \mathrm{H}_{3}$ plus acetylene also has an entrance channel barrier (14.9) and there is a downhill pathway to 1-dehydro-fulvene $(-55.0)$. 1-dehydro-fulvene can rearrange to 6 -dehydro-fulvene $(-60.3)$ by a 1,3-hydrogen shift over a barrier (4.0), which is still below the entrance channel barrier, from which rearrangement to phenyl radical can occur by a downhill pathway. Thus, both $n-\mathrm{C}_{4} \mathrm{H}_{3}$ and iso- $\mathrm{C}_{4} \mathrm{H}_{3}$ can react with acetylene to give phenyl radical with small barriers. (1) 1995 American Institute of Physics.
\end{abstract}

\section{INTRODUCTION}

The formation of the first aromatic ring (phenyl radical) is widely believed to be the rate limiting step in the formation of soot in hydrocarbon combustion. One mechanism for the formation of phenyl radical involves the stepwise addition of acetylenes. ${ }^{1}$ One possible pathway is the reaction of the vinyl radical with acetylene to give $\mathrm{C}_{4} \mathrm{H}_{3}$ followed by subsequent addition of another acetylene and ring closure to give phenyl radical. Another pathway to $\mathrm{C}_{4} \mathrm{H}_{3}$ is the reaction of vinylidene with acetylene to give vinylacetylene (buta-1-ene-3-yne), ${ }^{2}$ which can be converted to $\mathrm{C}_{4} \mathrm{H}_{3}$ (1- or 2-dehydro-buta-1-ene-3-yne) by abstraction of a hydrogen. Note that reaction of acetylene with itself does not occur by any low energy pathway, and vinylidene is $43 \mathrm{kcal} / \mathrm{mol}$ above acetylene; ${ }^{2,3}$ thus, the formation of vinylacetylene from two acetylenes requires a moderate activation energy. The structure and properties of $\mathrm{C}_{4} \mathrm{H}_{3}$ have been studied by $\mathrm{Ha}$ and $\mathrm{Gey}^{4}$ using PUMP4/6-31G** theory. They find two low-lying isomers 1-dehydro-buta-1-ene-3-yne $\left(n-\mathrm{C}_{4} \mathrm{H}_{3}\right)$ and 2-dehydro-buta-1-ene-3-yne (iso- $\mathrm{C}_{4} \mathrm{H}_{3}$ ). Iso- $\mathrm{C}_{4} \mathrm{H}_{3}$ is found to be $7.3 \mathrm{kcal} / \mathrm{mol}$ below $n-\mathrm{C}_{4} \mathrm{H}_{3}$ (including zero-point effects). Miller and Melius ${ }^{5}$ also noted that iso- $\mathrm{C}_{4} \mathrm{H}_{3}$ is thermodynamically more stable than $n-\mathrm{C}_{4} \mathrm{H}_{3}$ and thus most of the $\mathrm{C}_{4} \mathrm{H}_{3}$ should be in the form of the iso isomer even at combustion temperatures. Thus, it is of interest to study the reaction of both iso- $\mathrm{C}_{4} \mathrm{H}_{3}$ and $n-\mathrm{C}_{4} \mathrm{H}_{3}$ with acetylene, as discussed herein.

In Sec. II the technical details of the calculations are discussed, while Sec. III discusses the results, and Sec. IV concludes the paper.

\section{COMPUTATIONAL DETAILS}

Two different basis sets were used in these calculations. The stationary points were located with restricted HartreeFock (RHF) derivative calculations using the valence double zeta set of Dunning and Hay. ${ }^{6}$ The basis set for $\mathrm{C}$ is the $(9 s 5 p) /[3 s 2 p]$ basis and the $\mathrm{H}$ basis is $(4 s) /[2 s]$, i.e., the polarization functions are omitted. The subsequent internally contracted configuration interaction (ICCI) calculations were based on the RHF reference wave function and used the Dunning correlation consistent polarized valence double zeta basis set. ${ }^{7}$

The geometry optimizations used the SIRIUS/ABACUS system of programs, ${ }^{8}$ while the ICCI calculations were carried out with MOLPRO. ${ }^{9.10}$ All electrons were correlated except for the $\mathrm{C} 1 \mathrm{~s}$ like electrons. A multireference analog of the Davidson's correction ${ }^{11}$ was added to the ICCI energies and is denoted by $+Q$.

\section{DISCUSSION}

Table I(a) shows the computed ICCI energies of the stationary point structures for the reaction of iso- $\mathrm{C}_{4} \mathrm{H}_{3}$ with acetylene, while Table $I(b)$ shows the same information for the reaction of $n-\mathrm{C}_{4} \mathrm{H}_{3}$ with acetylene. The computed harmonic frequencies are given in Tables AIa and AIb of the PAPS material. ${ }^{12}$ The zero point effects were estimated as $1 / 2$ the sum of the harmonic frequencies and these are included in the relative energies, which are given in the last column of Tables I(a) and I(b). Finally, the Cartesian coordinates for the stationary points are given in Tables AIIa and AIIb of the PAPS material. 
TABLE I. (a) Computed energies for stationary points on the iso- $\mathrm{C}_{4} \mathrm{H}_{3}$ plus $\mathrm{C}_{2} \mathrm{H}_{2}$ surface. ${ }^{a}$ (b) Computed energies for stationary points on the $n-\mathrm{C}_{4} \mathrm{H}_{3}$ plus $\mathrm{C}_{2} \mathrm{H}_{2}$ surface. $^{\mathrm{b}}$

\begin{tabular}{lccr}
\hline \hline Geometry & ICCI $(\mathrm{ICCI}+Q+230)$. & ZPE & \multicolumn{1}{c}{$\Delta E$} \\
\hline & \multicolumn{2}{c}{$(\mathrm{a})$} & \\
reac & $-230.55140(-0.68546)$ & 0.07538 & 0.0 \\
sp1 & $-230.53032(-0.66848)$ & 0.08208 & 14.9 \\
min1 & $-230.61279(-0.74274)$ & 0.08799 & -28.0 \\
sp3 & $-230.60214(-0.73303)$ & 0.08570 & -23.4 \\
min3 & $-230.61250(-0.74239)$ & 0.08784 & -27.9 \\
sp6 & $-230.58635(-0.71908)$ & 0.08660 & -14.1 \\
min6 & $-230.65785(-0.78994)$ & 0.09223 & -55.0 \\
sp7 & $-230.55315(-0.68829)$ & 0.08452 & 4.0 \\
min7 & $-230.66583(-0.79763)$ & 0.09141 & -60.3 \\
sp8 & $-230.60110(-0.73403)$ & 0.08629 & -23.6 \\
min8 & $-230.61098(-0.74116)$ & 0.08799 & -27.0 \\
sp9 & $-230.52984(-0.66482)$ & 0.08295 & 17.7 \\
min9 & $-230.65985(-0.79156)$ & 0.09176 & -56.3 \\
sp10 & $-230.58726(-0.72029)$ & 0.08640 & -14.9 \\
min10 & $-230.61872(-0.74886)$ & 0.08678 & -32.6 \\
& & & \\
reactants & $-230.53798(-0.67101)$ & 0.07587 & 9.4 \\
sp2 & $-230.53009(-0.66357)$ & 0.08172 & 17.7 \\
min2 & $-230.60513(-0.73760)$ & 0.08829 & -24.6 \\
sp4 & $-230.59501(-0.72848)$ & 0.08596 & -20.4 \\
min4 & $-230.61098(-0.74116)$ & 0.08799 & -27.0 \\
sp5 & $-230.58919(-0.72947)$ & 0.08762 & -19.9 \\
min5 & $-230.72030(-0.85062)$ & 0.09409 & -91.9 \\
\hline \hline
\end{tabular}

Energies in a.u. except for $\Delta E$ which is the relative energy in $\mathrm{kcal} / \mathrm{mol}$ with respect to iso- $\mathrm{C}_{4} \mathrm{H}_{3}+\mathrm{C}_{2} \mathrm{H}_{2}$ including zero point energy.

Energiees in a.u. except for $\Delta E$ which is the relative energy in $\mathrm{kcal} / \mathrm{mol}$ with respect to iso- $\mathrm{C}_{4} \mathrm{H}_{3}+\mathrm{C}_{2} \mathrm{H}_{2}$ including zero point energy

The stationary points structures for the reaction of iso- $\mathrm{C}_{4} \mathrm{H}_{3}$ with acetylene are shown in Fig. 1, while those for the reaction of $n-\mathrm{C}_{4} \mathrm{H}_{3}$ with acetylene are shown in Fig. 2. Figure 3 shows the stationary point structures for the conver-

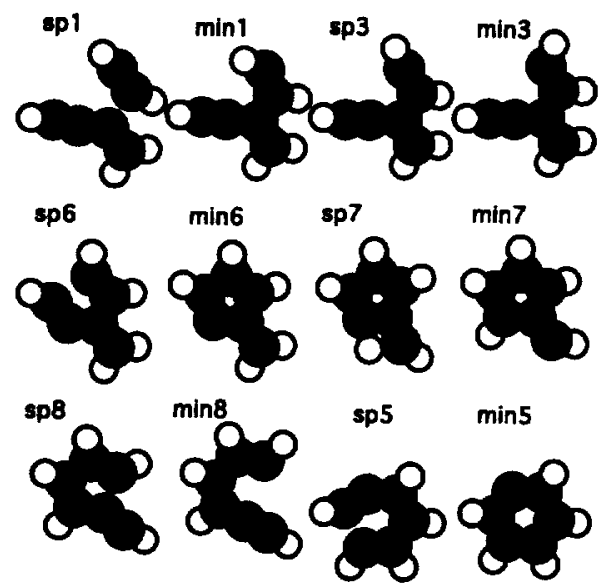

FIG. 1. Stationary point structures for the reaction of iso- $\mathrm{C}_{4} \mathrm{H}_{3}+\mathrm{C}_{2} \mathrm{H}_{2} \rightarrow \mathrm{C}_{6} \mathrm{H}_{5}$ (phenyl). The following nomenclature is used. Minl and $\min 3=1$-dehydro-3-methylene-penta-1-ene-4-yne. Min6=1-dehydrofulvene. Min7=6-dehydro-fulvene. Min8=1-dehydro-hexa-1,3-diene-5-yne $=$ Min4. Min5-phenyl radical. Structures designated by min are minima on the PES while structures designated by sp are saddle points. This convention is also used for Figs. 2 and 3.

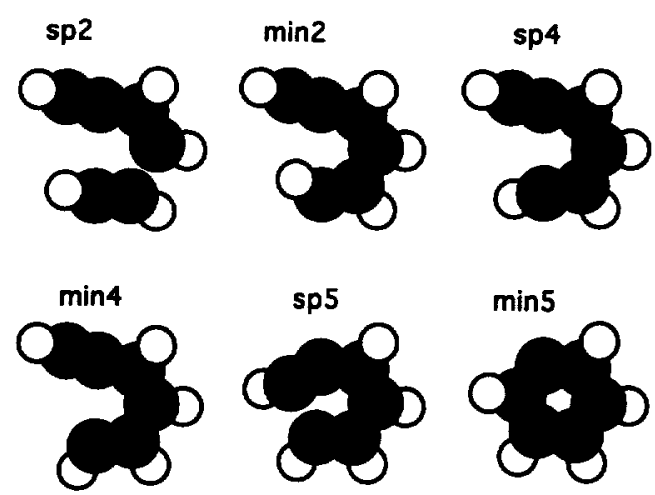

FIG. 2. Stationary point structures for the reaction of $n-\mathrm{C}_{4} \mathrm{H}_{3}+\mathrm{C}_{2} \mathrm{H}_{2} \rightarrow \mathrm{C}_{6} \mathrm{H}_{5}$ (phenyl). The following nomenclature is used. Min2 and min4 $=1$-dehydrohexa-1,3-diene-5-yne. Min5=phenyl radical.

sion of $\min 6$ to $\min 10$. (Here structures denoted by $\min$ are minima and structures denoted by $\mathrm{sp}$ are saddle points on the potential energy surface.) The energetics for these processes are shown in Figs. 4-6, respectively.

Consider first the entrance channel barriers for the reaction of $n-\mathrm{C}_{4} \mathrm{H}_{3}$ and iso- $\mathrm{C}_{4} \mathrm{H}_{3}$ with acetylene. $n-\mathrm{C}_{4} \mathrm{H}_{3}$ plus acetylene is $9.4 \mathrm{kcal} / \mathrm{mol}$ above iso- $\mathrm{C}_{4} \mathrm{H}_{3}$ plus acetylene, but the barrier to addition is $14.9 \mathrm{kcal} / \mathrm{mol}$ for iso- $\mathrm{C}_{4} \mathrm{H}_{3}$ plus acetylene and $8.3 \mathrm{kcal} / \mathrm{mol}$ for $n-\mathrm{C}_{4} \mathrm{H}_{3}$ plus acetylene. The higher barrier for iso- $\mathrm{C}_{4} \mathrm{H}_{3}$ plus acetylene probably results from larger nonbonding interactions between the two acetylenic groups, which come closer together in the iso- $\mathrm{C}_{4} \mathrm{H}_{3}$ plus acetylene case. This is reflected in the larger angle between the acetylenic groups in Fig. 1 as compared to Fig. 2.

Addition of $n-\mathrm{C}_{4} \mathrm{H}_{3}$ to acetylene leads to $\mathrm{min} 2$. Inversion about the $\mathrm{CH}$ group of $\mathrm{Cl}$ interconverts $\min 2$ and $\min 4$ with a barrier of $4.2 \mathrm{kcal} / \mathrm{mol}$. Min4 closes to min5 (phenyl radical) with only a small barrier. Thus, this reaction path leads to phenyl radical with no barrier other than the entrance channel addition barrier.

Addition of iso- $\mathrm{C}_{4} \mathrm{H}_{3}$ to acetylene leads to $\min 1$. Min1 is connected to $\min 3$ by $\operatorname{sp} 3$. The conversion from $\min 1$ to

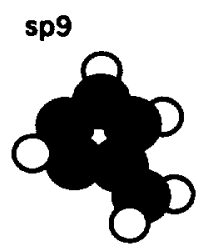

$\min 9$

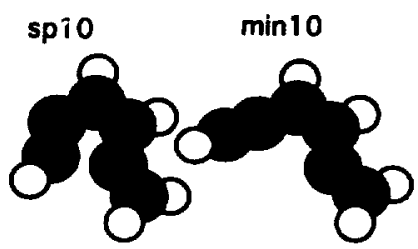

FIG. 3. Stationary point structures for the conversion of $\min 6$ to $\min 10$. The following nomenclature is used. Min9=2-dehydro-fulvene. Min $10=2$ dehydro-hexa-1,3-diene-5-yne 


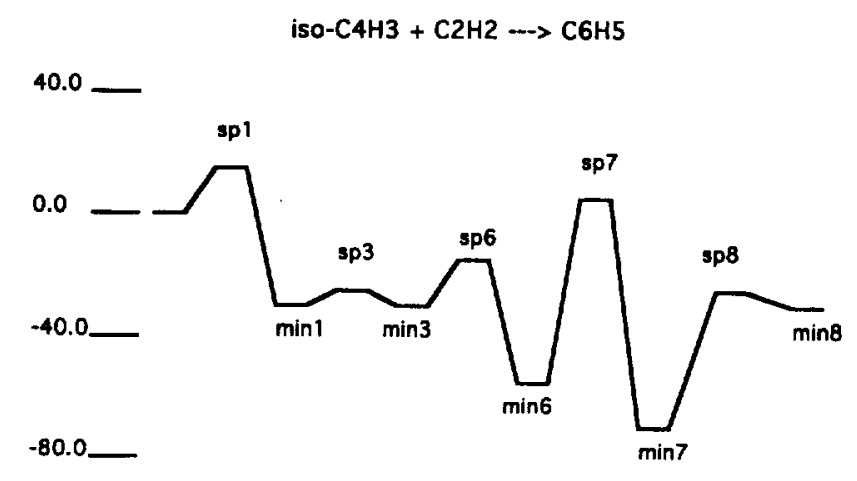

FIG. 4. Energetics from SDCI $+Q$ calculations with the cc-pVDZ basis set for iso- $\mathrm{C}_{4} \mathrm{H}_{3}+\mathrm{C}_{2} \mathrm{H}_{2} \rightarrow \mathrm{C}_{6} \mathrm{H}_{5}$ (phenyl).

$\min 3$ involves an inversion of the $\mathrm{CH}$ group about $\mathrm{Cl}$. This involves only a $4.6 \mathrm{kcal} / \mathrm{mol}$ barrier. Sp6 connects $\min 3$ to min6 (1-dehydro-fulvene). Sp7 is the saddle point for a 1,3hydrogen shift which connects min6 (1-dehydro-fulvene) to $\min 7$ (5-dehydro-fulvene). This process involves a large barrier; sp7 is $4 \mathrm{kcal} / \mathrm{mol}$ above iso- $\mathrm{C}_{4} \mathrm{H}_{3}$ plus acetylene, but still below the entrance channel saddle point, so this channel is energetically accessible. Min 7 can convert to $\min 8$ via sp8. Min8 is the same structure as min4 in the $n-C_{4} H_{3}$ plus acetylene reaction pathway. This can then convert to phenyl radical via a downhill pathway. Thus, both iso- $\mathrm{C}_{4} \mathrm{H}_{3}$ plus acetylene and $n-\mathrm{C}_{4} \mathrm{H}_{3}$ plus acetylene can give phenyl radical by pathways which involve no barriers, which are larger than the entrance channel addition barriers.

Sp9 is a saddle point connecting $\min 7$ to $\min 9$ (2dehydro-fulvene). $\mathrm{Sp} 9$ is $17.7 \mathrm{kcal} / \mathrm{mol}$ above iso- $\mathrm{C}_{4} \mathrm{H}_{3}$ plus acetylene, which is still below the entrance channel saddle point. However, $s p 9$ is a second order saddle point, i.e., it has two negative eigenvalues [see Table I(a)] one of which leads to symmetry breaking. Thus, there may be a lower energy path to min 9 if the symmetry is lowered. This point was not examined further in this work. Sp10 connects $\min 9$ to $\min 10$.

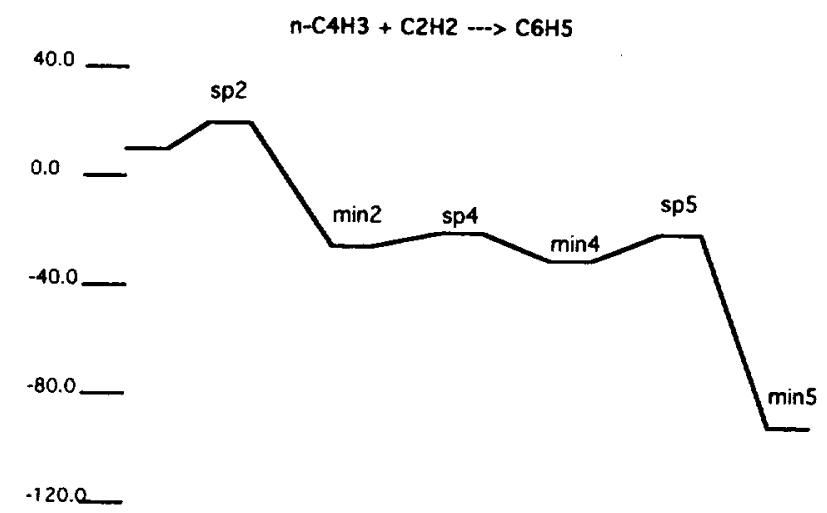

FIG. 5. Energetics from $\mathrm{SDCl}+Q$ calculations with the cc-pVDZ basis set for $n-\mathrm{C}_{4} \mathrm{H}_{3}+\mathrm{C}_{2} \mathrm{H}_{2} \rightarrow \mathrm{C}_{6} \mathrm{H}_{5}$ (phenyl).

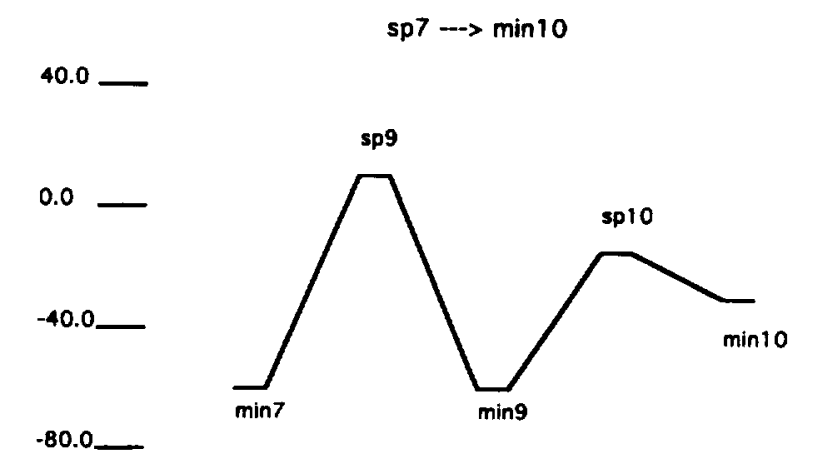

FIG. 6. Energetics from SDCI $Q$ calculations with the cc-pVDZ basis set for the conversion of $\min 6$ to $\min 10$.

\section{CONCLUSIONS}

The ring closure reactions of 1-dehydro-buta-1-ene-3yne $\left(n-\mathrm{C}_{4} \mathrm{H}_{3}\right)$ and 2-dehydro-buta-1-ene-3-yne (iso- $\mathrm{C}_{4} \mathrm{H}_{3}$ ) with acetylene have been studied using RHF plus singles and doubles CI plus a Davidson's correction with the Dunning cc-pVDZ basis set at stationary point structures determined by RHF derivative calculations using a polarized valence double zeta basis set. Zero-point effects were estimated based on the RHF harmonic frequencies.

$n-\mathrm{C}_{4} \mathrm{H}_{3}$ plus acetylene (9.4) has a small entrance channel barrier (17.7) (all energetics in parentheses are in $\mathrm{kcal} / \mathrm{mol}$ with respect to iso- $\mathrm{C}_{4} \mathrm{H}_{3}$ plus acetylene) and the subsequent closure steps leading to phenyl radical $(-91.9)$ are downhill with respect to the entrance channel barrier. Iso- $\mathrm{C}_{4} \mathrm{H}_{3}$ plus acetylene also has an entrance channel barrier (14.9) and there is a downhill pathway to 1 -dehydro-fulvene $(-55.0)$. 1-dehydro-fulvene can rearrange 6-dehydrofulvene $(-60.3)$ by a 1,3-hydrogen shift over a barrier (4.0), which is still below the entrance channel barrier. 6-dehydro-fulvene can rearrange to phenyl radical via 1-dehydro-hexa-1,3-diene-5yne $(-27.0)$. Thus, both $n-\mathrm{C}_{4} \mathrm{H}_{3}$ and iso- $\mathrm{C}_{4} \mathrm{H}_{3}$ can react with acetylene to give phenyl radical with small barriers.

With respect to iso- $\mathrm{C}_{4} \mathrm{H}_{3}$ plus acetylene, all of the open structures $(\min 1, \min 3, \min 8=\min 4$, and $\min 10)$ are from 27.0 to $32.6 \mathrm{kcal} / \mathrm{mol}$ lower, while the dehydro-fulvene structures (min6, $\min 7$, and $\min 9$ ) are from 55.0 to $60.3 \mathrm{kcal} / \mathrm{mol}$ lower, and the phenyl radical is $91.9 \mathrm{kcal} / \mathrm{mol}$ lower, respectively. Thus, since all these structures can interconvert with barriers below the entrance channel barrier, the ultimate product is expected to be phenyl radical, although other $\mathrm{C}_{6} \mathrm{H}_{5}$ species may still play a role as reactive intermediates.

\section{ACKNOWLEDGMENTS}

S.P.W. was supported by NASA Cooperative Agreement Number NCC2-478 to ELORET Institute and by NASA Contract No. NAS2-14031 to ELORET.

'I. Glassman, in Proceedings of the 22nd Symposium (International) on Combustion (The Combustion Institute, Pittsburgh, 1988).

${ }^{2}$ S. P. Walch and P. R. Taylor, J. Chem. Phys. (in press).

${ }^{3}$ M. M. Gallo, T. P. Hamilton, and H. F. Schaefer III, J. Am. Chem. Soc. 112, 8714 (1990).

${ }^{4}$ T-K Ha and E. Gey, J. Mol. Struct. 306, 197 (1994). 
${ }^{5}$ J. A. Miller and C. F. Mellius, Combust. Flame 91, 21 (1992).

${ }^{6}$ T. H. Dunning, Jr. and P. J. Hay, in Methods of Electronic Structure Theory, edited by H. F. Schaefer III (Plenum, New York, 1977).

${ }_{8}^{7}$ T. H. Dunning, Jr., J. Chem. Phys. 90, 1007 (1989).

${ }^{8}$ sIRIus is a MCSCF program written by $\mathrm{H}$. J. Jensen and $\mathrm{H}$. Agren and ABACUS is a MCSCF derivatives program written by T. Helgaker, $H$. J. Jensen, P. Jorenson, J. Olsen, and P. R. Taylor.

${ }^{9}$ H.-J. Werner and P. J. Knowles, J. Chem. Phys. 89, 5803 (1988).

${ }^{10}$ P. J. Knowles and H.J. Werner, Chem. Phys. Lett. 145, 514 (1988).
"S. R. Langhoff and E. R. Davidson, Int. J. Quantum Chem. 8, 61 (1974). ${ }^{12}$ See AIP document No. PAPS JCPSA6-103-8544-18 for 18 pages of tables of supplemental material. Order by PAPS number and journal reference from American Institute of Physics, Physics Auxilary Publication Service, Carolyn Gehlbach, 500 Sunnyside Boulevard, Woodbury, New York 11797-2999. Fax: 516-576-2223; e-mail: janis@aip.org. The price is $\$ 1.50$ for each microfiche ( 98 pages) or $\$ 5.00$ for photocopies of up to 30 pages, and $\$ 0.15$ for each additional page over 30 pages. Airmail additional. Make checks payable to the American Institute of Physics. 